\title{
A Review on Image Fusion Techniques
}

\author{
Sweta k. Shah ${ }^{1}$, Prof. D.U. Shah ${ }^{2}$ \\ Student, Department of Electronics and Communication, R.K. University, Rajkot, India ${ }^{1}$ \\ H.O.D. Department of Electronics and Communication, R.K. University, Rajkot, India ${ }^{2}$
}

\begin{abstract}
Image fusion is defined as the process of combining two or more different images into a new single image retaining important features from each image with extended information content. The result of image fusion is a new image which is more suitable for human and machine perception or further image processing tasks such as segmentation, feature extraction and object recognition. This paper presents review on some of the image fusion techniques i.e. simple average, simple maximum, PCA, DCT, DWT. Comparative study of all these techniques concludes that DWT is better approach.
\end{abstract}

Keywords: Image Fusion, Principal Component Analysis, Discrete Cosine Transform, Discrete Wavelet Transform

\section{INTRODUCTION}

Image Fusion is a mechanism to improve quality of information from a set of images. The fusion of images is often required for images acquired from different instrument modalities or capture techniques of the same scene or objects (like multi-sensor, multi-focus and multimodal images). For example, in multi-focus imaging one or more objects may be in-focus in a particular image, while other objects in the scene may be in focus in other images. For remotely sensed images, some have good spectral information whereas others have high geometric resolution. In the arena of biomedical imaging, two widely used modalities, namely the magnetic resonance imaging (MRI) and the computed tomography (CT) scan do not reveal identically every detail of brain structure. While CT scan is especially suitable for imaging bone structure and hard tissues, the MR images are much superior in depicting the soft tissues in the brain that play very important roles in detecting diseases affecting the skull base. These images are thus complementary in many ways and no single image is totally sufficient in terms of their respective information content. The advantages these images may be fully exploited by integrating the complementary features seen in different images through the process of image fusion that generates an image composed of features that are best detected or represented in the individual images. Important applications of the fusion of images include medical imaging, microscopic imaging, remote sensing, computer vision, and robotics. The first step toward fusion, which may be interpreted as a preprocessing step is the registration which brings down the constituting images to a common coordinate system as fusion of images is meaningful only when common objects in images have identical geometric configuration with respect to size, location and orientation in all the images. In the next step, the images are combined to form a single fused image through a judicious selection of proportions of different features from different images[19].

The process of image fusion must ensure that all salient information present in the source images are transferred to the composite image. Image fusion can be performed at three levels: pixel, object, and decision level. In this paper pixel level based image fusion process is presented to represent a fusion process generating a single combined image containing an additional truthful description than individual source image[6].

\section{IMAGE FUSION TECHNIQUES}

\section{A. Simple Average}

The regions of images that are in focus tend to be of higher pixel intensity. Thus this algorithms a simple way of obtaining an output image with all regions in focus. The value of the pixel $P(i, j)$ of each image is taken and added. This sum is then divided by 2 to obtain the average. The average value is assigned to the corresponding pixel of the output image which is given in equation (1). This is repeated for all pixel values[5].

$k(i, j)=\{X(i, j)+Y(i, j)\} / 2$

Where $X(i, j)$ and $Y(i, j)$ are two input images.

\section{B. Simple Maximum method:}

In this method, the resultant fused image is obtained by selecting the maximum intensity of corresponding pixels from both the input image[4].

$F(i, j)=\sum_{i=0}^{m} \sum_{j=0}^{n} \operatorname{maxA}(i, j) B(i, j)$

Where $A(i, j), B(i, j)$ are input images and $F(i, j)$ is fused image.

\section{Principal Component Analysis}

Principal component analysis (PCA) is a vector space transform often used to reduce multidimensional data sets to lower dimensions for analysis. PCA is the simplest and most useful of the true eigenvector-based multivariate analyses, because its operation is to reveal the internal structure of data in an unbiased way. If a multivariate dataset is visualized as a set of coordinates in a highdimensional data space ( 1 axis per variable), PCA supplies the user with a 2D picture, a shadow of this object when viewed from its most informative viewpoint. This dimensionally-reduced image of the data is the ordination diagram of the 1st two principal axes of the data, which when combined with metadata (such as gender, location etc) can rapidly reveal the main factors underlying the structure of data[1]. 
Basically Principal component analysis is a technique in $E$. which number of correlated variables are transformed into number of uncorrelated variables called principal components. A compact and optimal description of datasets is computed by PCA. The first principal component accounts for as much of the variance in the data as possible and each succeeding component accounts for as much of the remaining variance as possible. First principal component is taken to be along the direction with maximum variance. The second principal component is constrained to lie in the subspace perpendicular to the first within this subspace, this component points the direction of maximum variance. The third principal component is taken in the direction of maximum variance in the subspace perpendicular to the first two and so on. The PCA is also called as Karhunen-Loève transform or the Hotelling transform. The PCA does not have a fixed set of basis vectors like FFT, DCT and wavelet etc. and its basis vectors depend on the data set [2]. Image fusion process using PCA can be described as follow[4]:

$\checkmark$ From the input image matrices produce the column vectors.

$\checkmark$ Compute the covariance matrix of two column vectors formed before.

$\checkmark$ Compute the Eigen values and Eigen vectors of the covariance matrix.

$\checkmark \quad$ The column vector corresponding to the larger Eigen value is normalized by dividing each element with mean of Eigen vector.

$\checkmark$ Normalized Eigen vector value act as the weight values which are respectively multiplied with each pixel of the input images.

$\checkmark \quad$ The fused image matrix will be sum of the two scaled matrices .

\section{Discrete Cosine Transform}

A DCT is used to express a sequence of finite data points in terms of a sum of cosine functions oscillating at different frequencies. Discrete cosine transform (DCT) is an important transform extensively used in digital image processing. Large DCT coefficients are concentrated in the low frequency region. hence, it is known to have excellent energy compactness properties[3].

\section{Process Flow Diagram:}

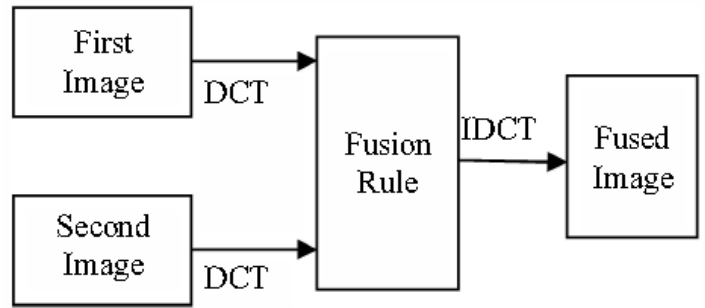

Fig. 1 Image Fusion Process using DCT

Images to be fused are divided into non-overlapping blocks of size $\mathrm{NxN}$ as shown in Fig-1. DCT coefficients are computed for each block and fusion rules are applied to get fused DCT coefficients. IDCT is then applied on the fused coefficients to produce the fused image/block[3]

\section{Discrete Wavelet Transform}

Wavelet transforms are multi-resolution image decomposition tool that provide a variety of channels representing the image feature by different frequency subbands at multi-scale. It is a famous technique in analyzing signals. When decomposition is performed, the approximation and detail component can be separated 2-D Discrete Wavelet Transformation (DWT) converts the image from the spatial domain to frequency domain. As shown in fig. 2 the image is divided by vertical and horizontal lines and represents the first-order of DWT, and the image can be separated with four parts those are LL1, LH1, HL1 and HH1[4].

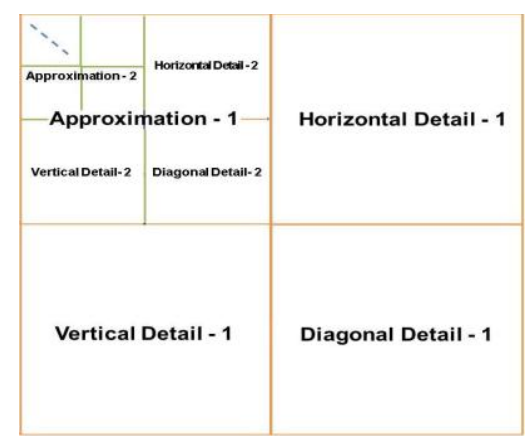

Fig 2. Wavelet Decomposition

General process of image fusion using DWT[4]:

Fig. 3 shows the process of image fusion using DWT.

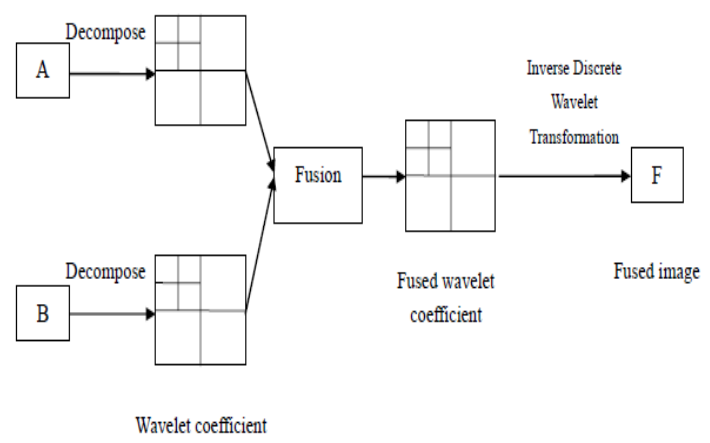

Fig 3. Wavelet based Image Fusion

Step 1. Implement Discrete Wavelet Transform on both the input image to create wavelet lower decomposition.

Step 2. Fuse each decomposition level by using different fusion rule.

Step 3. Carry Inverse Discrete Wavelet Transform on fused decomposed level, which means to reconstruct the image, while the image reconstructed is the fused image F.

\section{QUANTITATIVE IMAGE QUALITY METRICS}

Quality is a characteristic that measures perceived image degradation i.e., in comparison with ideal or perfect image. Evaluation forms an essential part in the development of image fusion techniques. It involves Full Reference where quality is measured in comparison with ideal image and No Reference Methods, which have no reference image[1]. Here we employ Full reference Methods and no reference method. 
A.

Full Reference Methods

Assumptions made in the following equations are as $\mathrm{A}$ is the image which is perfect, $B$ is the resultant image. $i, j$ is the pixel row and column index.

\section{a. $\quad$ Peak Signal to Noise Ratio (PSNR)}

PSNR is the ratio between the maximum possible power of a signal and the power of corrupting noise that affects the fidelity of its representation. The PSNR measure is given by[5]:-

$$
\operatorname{PSNR}(d b)=20 \log \frac{255 \sqrt{3 M N}}{\sqrt{\sum_{i=1}^{M} \sum_{j=1}^{N}\left(B^{\prime}(i, j)-B(i, j)\right)^{2}}}
$$

Where, $B$ - the perfect image, $B^{\prime}$ - the fused image to be assessed, $i$ - pixel row index, $j$ - Pixel column index, $\mathrm{M}$, N- No. of row and column.

\section{b. $\quad$ Structural Content(SC)}

The structural content measure used to compare two image in a number of small image patches the images have in common. The patches to be compared are chosen using 2D continuous wavelet which act as a low level corner detector. The large value of structural content means the image is of poor quality[7].

$$
S C=\frac{\sum_{i=1}^{M} \sum_{j=1}^{N}\left(A_{i j}\right)^{2}}{\sum_{i=1}^{M} \sum_{j=1}^{N}\left(B_{i j}\right)^{2}}
$$

\section{c. $\quad$ Entropy $(E N)$}

Entropy is an index to evaluate the information quantity contained in an image. If the value of entropy becomes higher after fusing, it indicates that the information increases and the fusion performances are improved. Entropy is defined as[5]:-

$E=\sum_{i=0}^{L-1} p_{i} \log _{2} p_{i}$

Where $\mathrm{L}$ is the total of grey levels, $p=\{p 0, p 1$ $p L-1\}$ is the probability distribution of each level .

\section{d. $\quad$ Mean Squared Error(MSE)}

Mean square error is measure of image quality index. Larger value of mean square error means that the image is of poor quality. The mathematical equation of MSE is given as[5]:

$$
M S E=\frac{1}{m n} \sum_{i=1}^{m} \sum_{j=1}^{n}\left(A_{i j-} B_{i j}\right)^{2}
$$

Where, $A$ - the perfect image, $B$ - the fused image to be assessed, $i$ - pixel row index, $j$ - pixel column index, $\mathrm{m}, \mathrm{n}-$ No. of row and column .

Normalized cross correlation are used to find out similarities between fused image and registered image is given by the following equation[5].

$N C C=\frac{\sum_{i=1}^{m} \sum_{j=1}^{n}\left(A_{i j} * B_{i j}\right)}{\sum_{i=1}^{m} \sum_{j=1}^{n}\left(A_{i j} B_{i j}\right)}$

\section{B. No Reference Method}

Fusion performance can be measured by the following fusion quality evaluation metrics' when we have no reference image: a. $\quad$ Spatial Frequency $(S F)$ :

Spatial Frequency indicates the overall activity in the fused image. The SF is computed as[2]:

Row Frequency:

$\mathrm{RF}=\sqrt{\frac{1}{M N} \sum_{x=0}^{M-1} \sum_{y=1}^{N-1}\left[I_{f}(x, y)-I_{f}(x, y-1)\right]^{2}}$

Column Frequency:

$\mathrm{CF}=\sqrt{\frac{1}{M N} \sum_{y=0}^{N-1} \sum_{x=1}^{M-1}\left[I_{f}(x, y)-I_{f}(x-1, y)\right]^{2}}$

Spatial Frequency $(\mathrm{SF})=\sqrt{R F^{2}+C F^{2}}$

Higher the SF means better performance.

b. Standard Deviation $(S D)$

Standard Deviation measures the contrast in the fused image. Fused image with high contrast would have high standard deviation[2].

$\mathrm{SD}=\sqrt{\frac{1}{M N} \sum_{y=0}^{N-1} \sum_{x=0}^{M-1}\left[I_{f}(x, y)-U_{I f}\right]^{2}}$

Where the mean is denoted as

$U_{I f}=\frac{1}{M N} \sum_{y=0}^{N-1} \sum_{x=0}^{M-1}\left|I_{f}(x, y)\right|$

\section{CONCLUSION}

In this paper, image fusion concept, some of the image fusion techniques are discussed and the image quality assessment parameters have been reviewed and determine the role of individual image quality assessment parameter to determine the quality of the fused image.Selection of proper fusion technique depends on the specific application.

The advantage of simple average technique is that it is the simplest method of image fusion, while its disadvantage is that this method does not give guarantee to have clear objects from the set of images. Simple maximum technique gives highly focused image output compared to average method, but its disadvantage is that it gives blurring effect which directly affect on the contrast of the image.

PCA technique has good spatial resolution, but produces spectral degradation. PCA and DCT can be used for applications which does not require high quality and precision.

DWT provides good quality fused images compared to Simple average, simple maximum, PCA and DCT techniques.

\section{REFERENCES}

[1] A. Sasi, L. Parameswaran, Sruthy S, "Image Fusion technique using DT-CWT" IEEE, 2013

[2] V.Naidu, J.Roal, "Pixel-level Image Fusion using Wavelets and Principal Component Analysis", Defence Science Journal,Vol.58, No.3, May 2008.

[3] R.Desale, S.Verma, "Study and Analysis of PCA,DCT \& DWT based Image Fusion Techniques",International Conference on Signal processing, Image Processing and Pattern Recognition 2013.

[4] K.Rani,R.Sharma, "Study of Different Fusion Algorithm", International journal of Emerging Technology and advanced Engineering, Vol.3, May 2013.

[5] D.Sahu, M.Parsai, "Different Image Fusion Techniques-A Critical Review", International Journal of Modern Engineering Research, Vol.2, Sep-Oct.2012 
[6] A.Ufade, M.Kawade, "Comparision of spatial domain and transform domain image fusion technique for restoration of blur images", International Conference on Recent Trends in engineering \& Technology-2013.

[7] S.Bedi, J.agarwal, P.Agarwal, "Image Fusion Techniques and Quality Assessment Parameters for Clinical Diagnosis: A Review", International Journal of Advanced Research in Computer and Communication Engineering, Vol. 2, Iss. 2, Feb. 2013.

[8] S.Krishnamoorthy, K.Soman, "Implementation and Comparative Study of Image Fusion Algorithms, International Journal of Computer Applications, Vol.9, Nov 2010.

[9] S.Li, B.Yang, J.Hu, "Information Fusion", Elsevier 2010.

[10] R.Singh, R.Dwivedi, S.Nagi, "Comparative Evaluation of DWT and DT-CWT for Image Fusion and De-noising", International Journal of Applied Information Systems, Vol 4, sept 2012.

[11] H.B. Mitchell. Image Fusion theories, techniques, and applications", Springer-Verlag Berlin Heidelberg, 2010.

[12] Y. Song, M. Li, Q. Li, and L. Sun, "A new wavelet based multifocus image fusion scheme and its application on optical microscopy," in Proceedings of IEEE Conference on Robotics and Biomimetics.

[13] F. Sadjadi, "Comparative Image Fusion Analysais", IEEE Computer Society Conference on Computer Vision and Pattern Recognition, Volume 3, Issue , 20-26 June 2005.

[14] J Zeng, A Sayedelahk, T Gilmore, P Frazier, M Chouika, "Review of Image Fusion Algorithms for Unconstrained Outdoor Scenes", Proceedings of the 8th International Conference on Signal Processing.

[15] C. Xydeas and V. Petrovic, "Objective pixel-level image fusion performance measure", Proceedings of SPIE, Vol. 4051, April 2000 .

[16] R. S. Blum, "The Cramer-Rao bound applied to image fusion," in Proceeding of the 8th International Conference on Information Fusion, Philadelphia, PA, July 2005.

[17] Zhang, Z. and Blum, R. A categorization of multiscaledecomposition-based image fusion schemes with a performance study for a digital camera application. Proceedings of the IEEE, Vol.87, pp. 1315-1326, 1999

[18] S. M. Mahbubur Rahman, M. Omair Ahmad and M.N.S Swamy, "Constant-based fusion of noisy image using discrete wavelet transform”, IET Image Process, Vol. 4 Iss.5, 2010.

[19] S.Bhargavi, Pavithra.C, "Fusion of two Images based on Wavelet Transform", Internatioal Journal of Innovative Research in Science, Engineering and Technology, Vol. 2 Iss. 5, May 2013.

[20] M.Pradnya,S.Ruikar, "Wavelet based Image Fusion Techniques ", International Conference on Intelligent Systems and Signal Processing 2013. 